\title{
The Prospective Application of Graphene Loaded Poly(4-vinphylpridine) Fibrous Scaffolds on the Dental Pulp Stem Cells Proliferation and Differentiation
}

\author{
Linxi Zhang ${ }^{1}$, Chung-Chueh Chang ${ }^{2}$, Marcia Simon $^{3}$, and Miriam Rafailovich ${ }^{1}$ \\ 1. Department of Materials Science and Engineering, Stony Brook University, Stony Brook, NY 11794, \\ USA \\ 2. Advanced Energy Research \& Technology Center, Stony Brook University, Stony Brook, NY 11794, \\ USA \\ ${ }^{3}$ Department of Oral Biology and Pathology, School of Dental Medicine, Stony Brook, NY 11794 , \\ USA
}

Dental Pulp Stem Cells (DPSCs) are a promising new approach for bone regeneration, since they are relatively easy to harvest without significant surgical access to the collection sites [1]. It is well established in the literature that DPSCs have the ability to differentiate along osteogenic, odontogenic or neural pathways. Studies have shown that the phenotype expressed is regulated by the microenvironment of the cells, or the stem cell niche where different biochemical cues, cell-cell interactions, cell-scaffold interactions are present [2]. The mechanical response of the substrate has been an important focus of many studies. However, most of these studies were performed on two dimensional structured substrates. In vivo the cellular environment is regulated by the extracellular matrix, which is fibrillar. Hence the essential questions arise as to the influence of the dimensions of the fibers on the cell differentiation. In order to study this aspect we developed a model based on polymer fibers, which serve to template the ECM proteins, and provide a structured scaffold where we can directly study the influence of topography on stem cell differentiation.

In this research, we have successfully fabricated three dimensional nano-sized fibrous scaffold of poly(4vinphylpridine) (P4VP) loaded with graphene nanoplatelets (GNPs) to mimic tissue's natural extracellular matrix and investigated its effect on DPSC behavior. Graphene-polymer composite materials have been popularized in tissue and engineering due to the excellent thermal, electrical and mechanical properties of graphene and previous studies have shown that graphene has ability to enhance the stem cell proliferation and mediate differentiation linages [3]. However, the dispersion of graphene in polymer matrix has always been challenging since the graphene tends to aggregate. In this study, the cost-effective technique, electrospinning, was applied to introduce the graphene into P4VP polymer matrix to fabricate the 3-D nano-sized scaffolds of GNPs-P4VP.

Electron Microscopes (EMs) were employed to characterize the structure and morphology of electrospun fibers. Figure 1A shows uniform defect-free fiber structures and excellent dispersion of graphene. The enhancement of physical properties was also revealed by Atomic force microscopy (AFM) on peak forceTUNA mode. To further investigate the effects on DPSCs behavior, DPSCs were cultured on these scaffolds and were incubated for up to 28days without the addition of any differentiation factors not normally present in vivo, such as dexamthosone. Laser Scanning Confocal Microscopy (LSCM) was applied to reveal the cell morphology on scaffolds after 7-day incubation as shown in figure 1B. The result showed that cells are well attached to the fibers and their actin filaments are well extended along the fiber orientation After 28-day incubation, SEM was used to characterize the surface of the scaffolds and a rich deposition of mineralized matrix was revealed as Figure $1 \mathrm{C}$ as shown. Energy-dispersive $\mathrm{x}$-ray spectroscopy (EDX) was also performed to analyze the elemental compositions of the matrix and the 
calcium-rich minerals covered only on fibers were identified suggesting the possibility of DPSCs osteogenic differentiation induced by the scaffolds. To confirm the possible osteogenic differentiation of DPSCs, immunocytochemistry was used to assess the state of the actin filaments, expression of osteocalcin (ocn) protein and location of the nuclei (Figure 1D). From figure 1D we find significant ocn expression in the cells, which is consistent with osteogenic differentiation and the depositions of hydroxyl apatite revealed by the SEM. Yet, despite differentiation, the cells are not dendritic, as has been reported on flat substrates, in the presence of dexamthsone. From the figure we see that they are confluent and form a tissue with well extended actin.

In conclusion we have demonstrated an efficient approach to manufacture the graphene loaded polymer 3-D scaffolds which are able to induce DPSC biomineralization and differentiation, without the addition of other factors such as dexamethasone, which is not a component normally present in vivo in the stem cell niche.

\section{References:}

[1] Riccardo d'Aquino et al, Stem Cell Reviews volume 4 (2008) p. 21-26.

[2] Akon Higuchi et al, Chemical Reviews volume 112 (2013), p. 4507-4540.

[3] Tapas R. Nayak et al, ACS Nano volume 5 (2011) p. 4670-4678.

[4] The authors acknowledge funding from the NSF-INSPIRE program and the ThINC core facility at Advanced Energy Research and Technology Center.
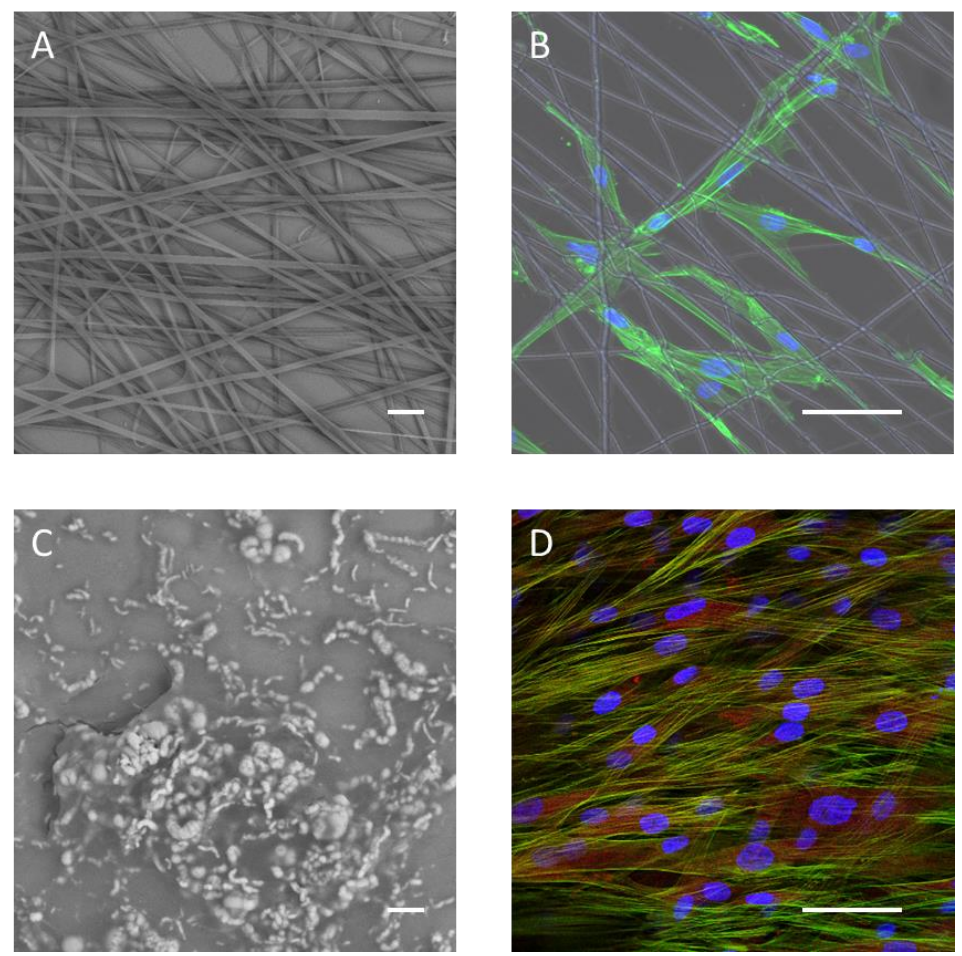

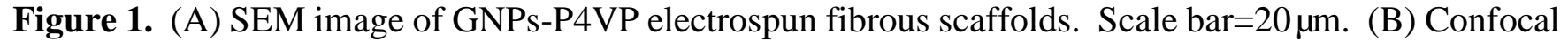
images of DPSCs on GNPs-P4VP scaffolds after 7-day incubation. Scale bar=75 $\mu \mathrm{m}$. (C) SEM image of calcium-rich minerals depositions on GNPs-P4VP scaffolds. Scale bar=20 $\mu \mathrm{m}$. (D) Confocal image of immunocytochemistry staining of DPSCs on GNPs-P4VP scaffolds. Scale bar $=75 \mu \mathrm{m}$. 